\title{
HISTORIA NATURAL DEL CÁNCER DE PRÓSTATA LOCALIZADO. DATOS PRELIMINARES DE PROGRESIÓN Y MORTALIDAD
}

\author{
M. LUJÁN GALÁN, A. PAÉZ BORDA, M.A. CABEZA RODRÍGUEZ*, \\ G.M. ESPINALES CASTRO, I. ROMERO CAGIGAL, C.A. ESCALERA ALMENDROS, \\ A. BERENGUER SÁNCHEZ
}

\begin{abstract}
Servicio de Urología. Hospital Universitario de Getafe. Madrid. *Servicio de Oncología Radioterápica. Hospital Universitario 12 de Octubre. Madrid.
\end{abstract}

Actas Urol Esp. 28 (5): 354-363, 2004

\section{RESUMEN \\ HISTORIA NATURAL DEL CÁNCER DE PRÓSTATA LOCALIZADO. DATOS PRELIMINARES DE PROGRESIÓN Y MORTALIDAD}

OBJETIVO: Conocer el impacto de la alternativa terapéutica y de otros factores sobre la historia natural del cáncer de próstata (CaP) localizado.

MÉTODOS: Pacientes con CaP clínicamente localizado sometidos a prostatectomía radical (PR), radioterapia (RT) u observación (OBS). Se calcularon las tasas de progresión bioquímica (PBQ) y clínica (PCL). Se evaluaron los efectos del tratamiento, del PSA al diagnóstico, de la presencia de tumor palpable y del score de Gleason mediante análisis KaplanMeier y test log-rank. Del mismo modo se estudiaron la mortalidad global y la cáncer específica.

RESULTADOS: Se estudiaron 228 pacientes (135 sometidos a PR, 46 a RT, y 47 a OBS). La mediana del tiempo de seguimiento fue de 2,5 años.

Cuarenta pacientes presentaron PBQ. La probabilidad de permanecer libre de PBQ a los 2 y 5 años fue de 76,8\% y 57,9\% respectivamente para la serie completa, $70,9 \%$ y $57,6 \%$ para PR, $100 \%$ y $100 \%$ para RT, y $87,1 \%$ y $47,2 \%$ para OBS $(\mathrm{p}=0,031)$. Diecinueve pacientes presentaron PCL, no observándose diferencia significativa respecto del tratamiento efectuado. Un score de Gleason pobremente diferenciado influyó en la probabilidad de presentar PCL ( $\mathrm{p}=0,022)$ y en la evolución a enfermedad metastásica $(\mathrm{p}<0,001)$. No se registró mortalidad cáncer-específica en la población estudiada.

CONCLUSIONES: El pronóstico a corto y medio plazo del cáncer de próstata localizado es, en términos de supervivencia, excelente. No obstante, algunos enfermos presentan un mayor riesgo de desarrollar enfermedad metastásica (Gleason pobremente diferenciado).

PALABRAS CLAVE: Cáncer de próstata. Historia natural. Supervivencia.

\section{ABSTRACT}

NATURAL HISTORY OF LOCALIZED PROSTATE CANCER. PRELIMINARY DATA ON PROGRESSION AND MORTALITY OBJECTIVE: To address the effect of therapy options and other factors on the natural history of localized prostate cancer $(\mathrm{PCa})$.

METHODS: Men with diagnosed clinically localized PCa who underwent radical prostatectomy (RP), radiotherapy (RT) or watchful waiting (WW). Rates of biochemical progression (BQP) and clinical progression (CLP) were calculated. The effects of therapy, initial PSA, presence of palpable tumor and Gleason score were assessed with Kaplan-Meier analysis and log-rank test. Similar methods were used to study overall and disease-specific survival.

RESULTS: A total of 228 patients were studied (135 underwent RP, $46 \mathrm{RT}$, and $47 \mathrm{WW}$ ). Median followup time was 2.5 years.

Forty patients presented with BQP. The probability of being free from BQP after 2 and 5 years was $76.8 \%$ and $57.9 \%$ respectively for the whole population, $70.9 \%$ and $57.6 \%$ for RP patients, $100 \%$ and $100 \%$ for RT, and $87.1 \%$ and $47.2 \%$ for WW ( $\mathrm{p}=0.031)$. Nineteen patients presented with CLP, with no significant differences with regard to therapy option. A poorly differentiated Gleason score favoured the probability of presenting with CLP $(\mathrm{p}=0.022)$ and shift to metastatic disease $(\mathrm{p}<0.001)$. No cancer-specific mortality was recorded in the studied population.

CONCLUSIONS: Short and medium term prognosis is excellent for localized prostate cancer in terms of survival. Nevertheless, some patients show a higher risk of progressing to metastatic disease (poorly differentiated Gleason score).

KEY WORDS: Prostate cancer. Natural history. Survival. 
$\mathrm{E}$ 1 cáncer de próstata $(\mathrm{CaP})$ constituye un relevante problema de salud. En el año 2000 fallecieron por esta causa en España 5.456 varones, situándose como la segunda causa de fallecimiento por neoplasia en varones por detrás del cáncer broncopulmonar, y por delante del colorrectal ${ }^{1}$. En nuestro ámbito (área sanitaria 10 Insalud, datos no publicados) las tasas de incidencia bruta y ajustada a la población mundial en el año 2002 fueron 58,8 y 41,2 casos / 100.000 varones respectivamente, y las tasas de mortalidad cáncerespecífica bruta y ajustada a la población mundial durante el mismo periodo fueron 4,9 y 3,5 fallecimientos / 100.000 varones respectivamente.

No obstante, cuando esta enfermedad se presenta en un estadio confinado a la glándula (localizado) y la esperanza de vida del enfermo se considera óptima, se recomiendan habitualmente tratamientos con intención curativa ${ }^{2}$. Los más empleados son la prostatectomía radical (PR) y la radioterapia prostática (RT) en distintas modalidades. Independientemente del tratamiento elegido, el presentar un estadio localizado supone un dato favorable a la hora de predecir la historia natural de la enfermedad ${ }^{3}$.

La vigilancia $\mathrm{u}$ observación $\sin$ tratamiento activo (OBS) ha sido considerada por varios autores como una opción a tener en cuenta en el abordaje del CaP localizado ${ }^{4-6}$, sobre todo en pacientes a partir de determinadas edades y con tumores bien diferenciados. No obstante, aunque se trata de tumores de crecimiento lento en la mayoría de ocasiones, existe riesgo de morir por esta enfermedad a largo plazo ${ }^{7}$. Dicho riesgo se acentúa en tumores con score de Gleason entre 7 y $10^{8}$.

El objetivo del presente trabajo es comparar la historia natural del CaP en cuanto a progresión y supervivencia en una serie no randomizada de pacientes con CaP clínicamente localizado sometidos a tratamiento con intención curativa (prostatectomía radical o radioterapia prostática), o bien incluidos en un programa de vigilancia.

\section{MATERIAL Y MÉTODOS}

A partir de una base de datos con 645 pacientes con $\mathrm{CaP}$ atendidos en nuestro Centro desde su creación en 1991 hasta final de 2002 (con fecha de diagnóstico anterior o comprendida en este periodo), se han seleccionado de modo retrospectivo los que fueron sometidos a una de las siguientes alternativas: PR, RT u OBS. El protocolo de nuestro Centro contempla ofrecer tratamiento con intención curativa (PR o RT) a todos los pacientes con CaP localizado y esperanza de vida superior a los 10 años (ausencia de patología concomitante severa y edad no superior a 70 años), recomendándose OBS al resto de pacientes. En el presente estudio no existió randomización alguna, dejando al paciente la decisión última sobre la opción terapéutica tras recibir información completa (recomendación según nuestro protocolo, así como ventajas e inconvenientes de cada opción). Los pacientes sometidos a PR fueron intervenidos en nuestro Centro por vía retropúbica. Los casos en que se indicó RT fueron remitidos a una Unidad de referencia ajena a nuestro Centro donde se suministró RT convencional a dosis estándar, realizándose posteriormente el seguimiento en nuestro Hospital.

Para el presente trabajo se excluyeron los casos de $\mathrm{CaP}$ que no presentaron estadio clínico localizado. Otros criterios de exclusión fueron una edad superior a los 70 años al diagnóstico, o la presencia de patología con potencial influencia sobre la expectativa de vida.

Se llevó a cabo un análisis descriptivo de las características al diagnóstico de los pacientes mencionados, teniendo en cuenta la edad, el valor de PSA sérico, el tacto rectal (TR) y el score de Gleason. Se estableció comparación entre los tres grupos terapéuticos con respecto a las variables mencionadas (test de Kruskal-Wallis para variables numéricas y test del Chi-cuadrado para categóricas).

Posteriormente se analizó la historia natural de la enfermedad (progresión y supervivencia). Se consideraron evaluables para la progresión los pacientes de todos los grupos con un tiempo de seguimiento mínimo de tres meses.

Se definió progresión bioquímica (-PBQ- o de PSA) el presentar valores de PSA total por encima de $0,2 \mathrm{ng} / \mathrm{ml}$ tras la PR, o bien 3 incrementos consecutivos de PSA ${ }^{9}$ en los grupos RT y OBS (en estos dos grupos se excluyeron por tanto los casos con menos de 4 determinaciones de PSA).

La progresión clínica (PCL) se definió como cualquier evolución a un estadio clínico menos favorable en cualquiera de las categorías $\mathrm{T}, \mathrm{N}$ o 
$\mathrm{M}$, incluyendo hematuria por invasión vesical, retención urinaria en pacientes con $\mathrm{CaP}$ localmente avanzado, u obstrucción ureteral por el tumor.

Para el cálculo de la tasa de progresión ajustada (TPA) se tuvo en cuenta el tiempo de seguimiento de cada grupo estudiado, de acuerdo con la siguiente expresión:

$\mathrm{TPA}=\left(\mathrm{n}^{\mathrm{o}}\right.$ de eventos $\left.\mathrm{x} 100\right) / \sum$ tiempo seguimiento varones evaluables (años).

(Las unidades de TPA se ofrecen en $n^{\circ}$ progresiones / 100 varones $\mathrm{x}$ año).

Se evaluó el efecto de determinadas variables (el tratamiento efectuado, el nivel de PSA al diagnóstico, la presencia de tumor palpable y el score de Gleason) sobre el tiempo hasta la aparición de PBQ, PCL, o hasta cualquier tipo de progresión (PBQ o PCL indistintamente). Para evaluar el efecto de las variables a estudio sobre el tiempo hasta la progresión se utilizaron las curvas de Kaplan-Meier, que se compararon por medio del test log-rank.

Para valorar el efecto de cada variable en presencia del resto de covariables se intentó la realización de un análisis multivariante (mediante un modelo de Cox) y el cálculo de las razones de riesgo para cada variable predictiva. No obstante, dicho análisis no pudo ser llevado a cabo porque las variables estudiadas no presentaron riesgos proporcionales.

Por último, se estudiaron la mortalidad global y la cáncer específica, realizando un análisis de supervivencia como el mencionado arriba. Los datos de mortalidad se obtuvieron del Servicio de Informática de nuestro Centro, que dispone de codificación de los fallecimientos ocurridos en el Hospital, con posterior acceso a las Historias Clínicas para su verificación. La mortalidad extrahospitalaria fue investigada tras cruzar nuestra base de datos con la del Instituto Nacional de Estadística (INE).

El nivel de significación estadística se estableció para una $\mathrm{p}<0,05$. El análisis de los datos se llevó a cabo mediante un PC con los paquetes informáticos Microsoft Access 97 (Microsoft Corp., Chicago IL, USA) y SPSS 8.0 (SPSS Inc., Chicago IL, USA).

\section{RESULTADOS}

Características de los pacientes estudiados

Un total de 228 pacientes fueron finalmente incluidos en el estudio. De ellos 135 fueron sometidos a PR, 46 a RT, y 47 a OBS. En la Tabla I se

\section{TABLA I}

CARACTERÍSTICAS CLÍNICAS (AL DIAGNÓSTICO) DE LOS TUMORES ESTUDIADOS

\begin{tabular}{|c|c|c|c|c|c|c|c|c|}
\hline & & & \multicolumn{4}{|c|}{ Tratamiento efectuado } & & \\
\hline & & & Total & PR & $\mathrm{RT}$ & OBS & \multicolumn{2}{|c|}{ Análisis comparativo } \\
\hline & & & $\mathrm{n}=228$ & $\mathrm{n}=135$ & $\mathrm{n}=46$ & $\mathrm{n}=47$ & Test & Valor $\mathrm{p}$ \\
\hline $\begin{array}{l}\text { Edad } \\
\text { (años) }\end{array}$ & \multicolumn{2}{|c|}{$\begin{array}{l}\text { M (Mín-Máx) } \\
\text { m (EEM) }\end{array}$} & $\begin{array}{l}62(41-70) \\
61,6(0,37)\end{array}$ & $\begin{array}{l}61(41-70) \\
60,4(0,48)\end{array}$ & $\begin{array}{c}61,5(53-70) \\
62,2(0,83)\end{array}$ & $\begin{array}{l}66(49-70) \\
64,4(0,70)\end{array}$ & $\begin{array}{l}\text { Kruskal- } \\
\text { Wallis }\end{array}$ & $<0,001$ \\
\hline $\begin{array}{l}\text { PSA } \\
(\mathrm{ng} / \mathrm{ml})\end{array}$ & \multicolumn{2}{|c|}{$\begin{array}{l}\text { M (Mín-Máx) } \\
\text { m (EEM) }\end{array}$} & $\begin{array}{c}7(1-119) \\
9,61(0,73)\end{array}$ & $\begin{array}{l}7(1,1-31,1) \\
8,33(0,47)\end{array}$ & $\begin{array}{c}8,35(1,39-119) \\
15,1(3,05)\end{array}$ & $\begin{array}{c}5,2(1-44,2) \\
7,99(1,20)\end{array}$ & $\begin{array}{l}\text { Kruskal- } \\
\text { Wallis }\end{array}$ & 0,002 \\
\hline $\begin{array}{l}\text { TR } \\
\text { Anormal }\end{array}$ & \multicolumn{2}{|c|}{ Frecuencia (\%) } & $79(34,6 \%)$ & $49(36,3 \%)$ & $18(39,1 \%)$ & $12(25,5 \%)$ & $\begin{array}{c}\text { Chi } \\
\text { cuadrado }\end{array}$ & 0,317 \\
\hline \multirow{4}{*}{$\begin{array}{l}\text { Score } \\
\text { Gleason }\end{array}$} & \multirow{4}{*}{$\begin{array}{l}\text { Frecuencia } \\
(\%)\end{array}$} & $2-4$ & $54(25,4 \%)$ & $30(23,3 \%)$ & $8(18,6 \%)$ & $16(39,0 \%)$ & \multirow{4}{*}{$\begin{array}{c}\text { Chi } \\
\text { cuadrado }\end{array}$} & \multirow{4}{*}{0,258} \\
\hline & & $5-6$ & $106(49,8 \%)$ & $66(51,2 \%)$ & $24(55,8 \%)$ & $16(39,0 \%)$ & & \\
\hline & & 7 & $38(17,8 \%)$ & $26(20,2 \%)$ & $7(16,3 \%)$ & $5(12,2 \%)$ & & \\
\hline & & $8-10$ & $15(7 \%)$ & $7(5,4 \%)$ & $4(9,3 \%)$ & $4(9,8 \%)$ & & \\
\hline
\end{tabular}

Mín-Máx: Valores mínimo y máximo.

M: Mediana.

m: media.

EEM: Error estándar de la media. 
ofrecen las características clínicas de los tumores incluidos. Se observó una tendencia significativa hacia una mayor edad en el grupo sometido a OBS respecto a los grupos de terapia activa $(\mathrm{p}<0,001)$. Asimismo se evidenció una mayor cifra de PSA total al diagnóstico en los varones que optaron por RT respecto de los otros dos grupos $(\mathrm{p}=0,002)$. No se observaron diferencias entre los grupos estudiados con respecto a la proporción de cáncer palpable ni al score de Gleason en la biopsia.

Un total de 204 pacientes $(89,5 \%)$ disponían de un tiempo de seguimiento superior a 3 meses. En este grupo dicho tiempo osciló entre los 0,3 y los 17,2 años, con una mediana de 2,5 años y una media de 2,9 años (error estándar de la media -EEM- 0,17).

\section{Progresión bioquímica}

Para el análisis de la PBQ fueron evaluables 162 pacientes. De ellos, $40(24,7 \%)$ presentaron PBQ (33 en el grupo PR, ninguno en el RT y 7 en el OBS).

La probabilidad de permanecer libre de PBQ a los 2 y 5 años fue de $76,8 \%$ y $57,9 \%$ respectivamente para la serie completa, $70,9 \%$ y $57,6 \%$ para PR, $100 \%$ y $100 \%$ para RT, y $87,1 \%$ y $47,2 \%$ para OBS. La diferencia entre estas probabilidades alcanzó el nivel marcado de significación estadística (log rank, p = 0,031, Tabla IIa).
En las Figuras la y lb se muestran las curvas de probabilidad de presentar PBQ. Ninguno de los pacientes evaluados en el grupo RT presentó PBQ tal y como ha sido definida en este estudio. No se evidenciaron diferencias en cuanto a la probabilidad de PBQ al tener en cuenta los niveles de PSA, el presentar tumor palpable, o el score de Gleason (Tablas IIb, IIc y IId).

\section{Progresión clínica}

De los 204 pacientes evaluables, 19 presentaron PCL (9,3\%). En las Tablas IIa, IIb, IIc y IId y en la Figura 2 se ofrecen resultados detallados.

Las tasas de permanencia libre de PCL a los 2 y 5 años fueron de $91,3 \%$ y $85,0 \%$ respectivamente para toda la serie, $97,7 \%$ y $88,8 \%$ para PR, $76,8 \%$ y $76,8 \%$ para RT, y $84,5 \%$ y $84,5 \%$ para OBS ( $p=0,109)$. El grupo de pacientes con mayor tendencia a la PCL fue RT (6 pacientes progresaron de 20 evaluables, esto es 6,30 eventos / 100 varones $\mathrm{x}$ año). No se encontraron diferencias en cuanto a la PCL cuando se tuvieron en cuenta datos como el nivel de PSA o el dato de tumor palpable (Tablas IIb y IIc). Por el contrario, un score de Gleason elevado influyó de modo significativo en la probabilidad de presentar PCL ( $p=0,022$, Tabla IId).

De los 19 pacientes que presentaron PCL, 7 lo hicieron en forma de enfermedad metastásica (3,4\% del total evaluable). Tres de ellos formaron parte del grupo PR, 1 del RT y 3 del OBS. Ni el

\section{TABLA IIa}

PROBABILIDAD DE PROGRESIÓN DE LA ENFERMEDAD (ANÁLISIS KAPLAN-MEIER). RESULTADOS SEGÚN TRATAMIENTO

\begin{tabular}{|c|c|c|c|c|c|c|}
\hline & & \multicolumn{4}{|c|}{ Tratamiento efectuado } & \multirow{2}{*}{$\begin{array}{l}\text { Log-rank } \\
\text { (p) }\end{array}$} \\
\hline & & Toda la serie & PR & RT & OBS & \\
\hline $\begin{array}{l}\text { Progresión } \\
\text { Bioquímica }\end{array}$ & \multirow{4}{*}{$\begin{array}{l}\text { TPA (eventos / } 100 \text { varones x año) } \\
\% \text { libre de progresión a los } 2 \text { años } \\
\% \text { libre de progresión a los } 5 \text { años }\end{array}$} & $\begin{array}{c}9,22 \\
76,8 \% \\
57,9 \%\end{array}$ & $\begin{array}{l}11,54 \\
70,9 \% \\
57,6 \%\end{array}$ & $\begin{array}{l}\text { No eventos } \\
100 \% \\
100 \%\end{array}$ & $\begin{array}{c}8,83 \\
87,1 \% \\
47,2 \%\end{array}$ & 0,031 \\
\hline Progresión clínica & & $\begin{array}{c}3,28 \\
91,3 \% \\
85,0 \%\end{array}$ & $\begin{array}{c}2,11 \\
97,7 \% \\
88,8 \%\end{array}$ & $\begin{array}{c}6,30 \\
76,8 \% \\
76,8 \%\end{array}$ & $\begin{array}{c}4,72 \\
84,5 \% \\
84,5 \%\end{array}$ & 0,109 \\
\hline $\begin{array}{l}\text { Progresión a } \\
\text { enfermedad } \\
\text { metastásica }\end{array}$ & & $\begin{array}{c}1,20 \\
96,0 \% \\
94,5 \%\end{array}$ & $\begin{array}{c}0,79 \\
98,6 \% \\
96,5 \%\end{array}$ & $\begin{array}{c}1,05 \\
93,1 \% \\
93,1 \%\end{array}$ & $\begin{array}{c}2,85 \\
89,6 \% \\
89,6 \%\end{array}$ & 0,310 \\
\hline $\begin{array}{l}\text { cualquier tipo de } \\
\text { progresión }\end{array}$ & & $\begin{array}{l}10,39 \\
72,9 \% \\
55,7 \%\end{array}$ & $\begin{array}{c}11,54 \\
70,9 \% \\
57,6 \%\end{array}$ & $\begin{array}{c}6,30 \\
76,8 \% \\
76,8 \%\end{array}$ & $\begin{array}{c}10,99 \\
73,9 \% \\
44,5 \%\end{array}$ & 0,470 \\
\hline
\end{tabular}

TPA = Tasa de progresión ajustada por tiempo de seguimiento. 


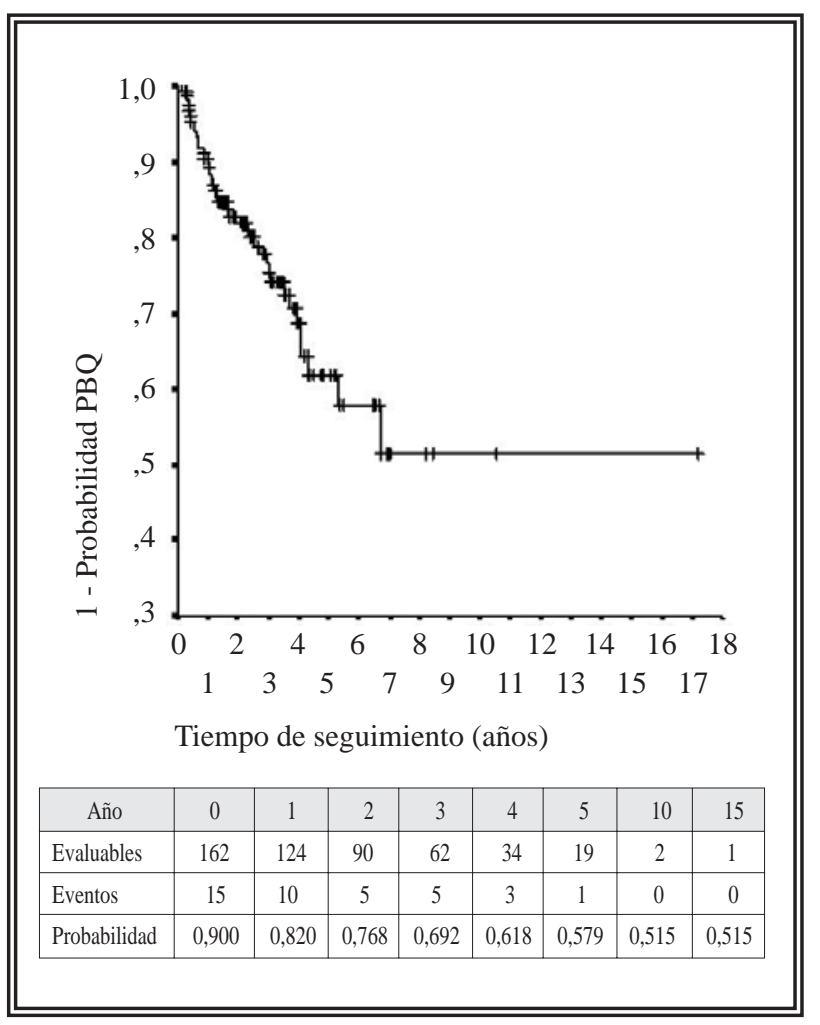

FIGURA 1a. Análisis Kaplan-Meier donde se ofrece la evolución de toda la serie en cuanto al evento progresión bioquimica.

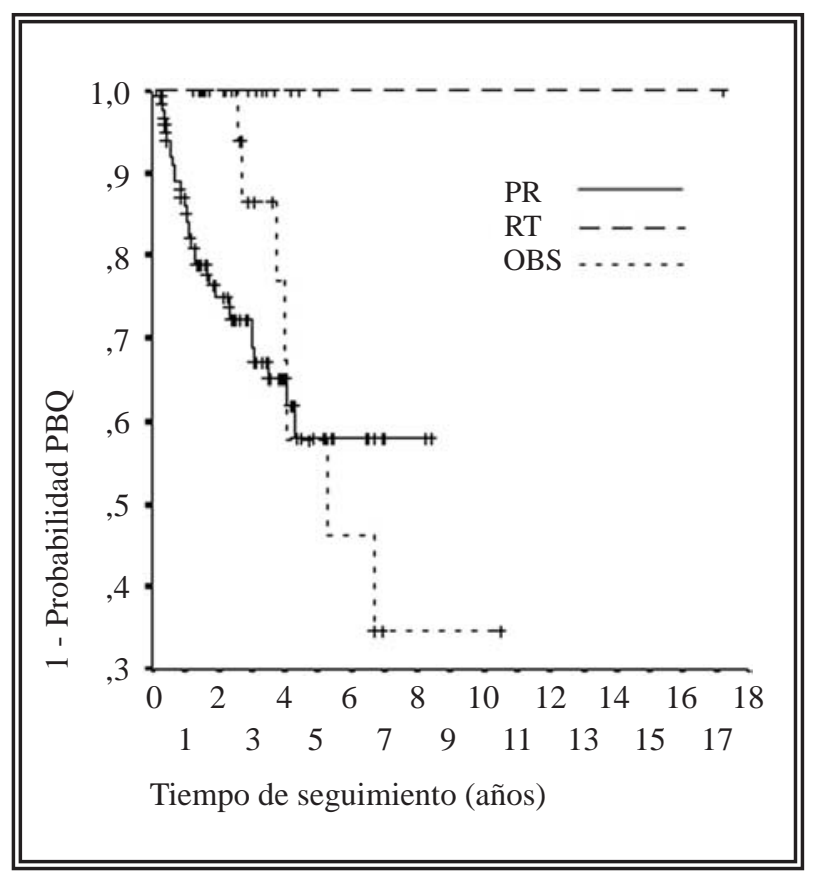

FIGURA 1b. Análisis actuarial donde se evalúa la diferencia en cuanto a progresión bioquímica entre los grupos de tratamiento estudiados $(P R=$ prostatectomía radical, $\mathrm{RT}$ = radioterapia, $\mathrm{OBS}$ = observación). Test log-rank $p=0,031$. tratamiento realizado, ni el nivel de PSA inicial, ni ser un tumor palpable influyeron de modo significativo en la evolución a CaP metastásico. Unicamente un score de Gleason pobremente diferenciado (Tabla IId) fue factor determinante para dicha evolución $(\mathrm{p}<0,001)$.

Cualquier tipo de progresión (PBQ o PCL indistintamente)

Un total de 204 pacientes fueron evaluables para el análisis de la progresión de cualquier tipo. Un total de 50 progresaron $(24,5 \%)$.

Las tasas de permanencia libre de PBQ o PCL a los 2 y 5 años fueron de $72,9 \%$ y $55,7 \%$ respectivamente para toda la serie (Fig. 3), 70,9\% y $57,6 \%$ para PR, $76,8 \%$ y $76,8 \%$ para RT, y $73,9 \%$ y $44,5 \%$ para OBS ( $\mathrm{p}=0,470$, Tabla IIa). Tampoco se observó relación entre las variables estudiadas y la probabilidad de progresión de cualquier tipo (Tablas IIb, IIc y IId).

\section{Supervivencia global y cáncer-específica}

Hemos detectado un total de 6 fallecimientos en la población estudiada $(2,6 \%)$. De ellos, 1 correspondió al grupo PR, 2 al RT y 3 al OBS. La supervivencia global media de toda la serie fue de 16,4 años (EEM 0,31). La tasas de supervivencia global a los 2 y 5 años fueron de 96,5\% y 95,1\% respectivamente en toda la serie (Fig. 4), 99,2\% y 99,2\% en el grupo PR, $96,5 \%$ y $85,8 \%$ en el RT, y $87,7 \%$ y $87,7 \%$ en el OBS. Esta ligera ventaja en cuanto a supervivencia global que ofreció la PR respecto a las otras dos opciones estudiadas alcanzó rango significativo (log-rank $\mathrm{p}=0,034$ ). Ninguno de los otros factores (edad, valor de PSA inicial, tumor palpable o score de Gleason -Tabla III- mostró influencia sobre la supervivencia global.

No se registraron muertes por $\mathrm{CaP}$ en la serie estudiada (ausencia de mortalidad cáncer-específica).

\section{DISCUSIÓN}

$\mathrm{El}$ presente trabajo pretende evaluar la historia natural, en términos de progresión y supervivencia, del CaP localizado abordado mediante las alternativas más habituales en la práctica urológica: PR, RT y OBS. En cualquier caso, es necesario recordar que se trata de resultados preliminares debido al limitado tiempo de seguimiento de la muestra estudiada (mediana de 2,5 años). 
TABLA IIb

PROBABILIDAD DE PROGRESIÓN DE LA ENFERMEDAD (ANÁLISIS KAPLAN-MEIER). RESULTADOS SEGÚN CIFRAS DE PSA AL DIAGNÓSTICO

\begin{tabular}{|c|c|c|c|c|c|c|c|}
\hline & & \multicolumn{5}{|c|}{ PSA al diagnóstico (ng/ml) } & \multirow{2}{*}{$\begin{array}{l}\text { Log-rank } \\
\text { (p) }\end{array}$} \\
\hline & & Toda la serie & $<4$ & $\geq 4 \mathrm{y}<7$ & $\geq 7 y<10$ & $>10$ & \\
\hline $\begin{array}{l}\text { Progresión } \\
\text { bioquímica }\end{array}$ & \multirow{4}{*}{$\begin{array}{l}\text { TPA (eventos / } 100 \text { varones x año) } \\
\% \text { libre de progresión a los } 2 \text { años } \\
\% \text { libre de progresión a los } 5 \text { años }\end{array}$} & $\begin{array}{c}9,22 \\
76,8 \% \\
57,9 \% \\
\end{array}$ & $\begin{array}{c}6,11 \\
89,5 \% \\
44,0 \%\end{array}$ & $\begin{array}{c}7,53 \\
79,9 \% \\
67,2 \%\end{array}$ & $\begin{array}{c}7,23 \\
78,5 \% \\
66,4 \% \\
\end{array}$ & $\begin{array}{l}15,76 \\
65,1 \% \\
50,5 \%\end{array}$ & 0,147 \\
\hline $\begin{array}{l}\text { Progresión } \\
\text { clinica }\end{array}$ & & $\begin{array}{c}3,28 \\
91,3 \% \\
85,0 \%\end{array}$ & $\begin{array}{c}1,23 \\
94,9 \% \\
94,9 \%\end{array}$ & $\begin{array}{c}2,16 \\
93,8 \% \\
83,4 \%\end{array}$ & $\begin{array}{c}4,51 \\
92,0 \% \\
85,2 \%\end{array}$ & $\begin{array}{c}5,39 \\
84,9 \% \\
80,2 \%\end{array}$ & 0,263 \\
\hline $\begin{array}{l}\text { Progresión a } \\
\text { enfermedad } \\
\text { metastásica }\end{array}$ & & $\begin{array}{c}1,20 \\
96,0 \% \\
94,5 \% \\
\end{array}$ & $\begin{array}{l}\text { No eventos } \\
100 \% \\
100 \%\end{array}$ & $\begin{array}{c}1,08 \\
95,3 \% \\
95,3 \% \\
\end{array}$ & $\begin{array}{c}0,90 \\
96,8 \% \\
96,8 \% \\
\end{array}$ & $\begin{array}{c}2,39 \\
93,7 \% \\
88,5 \% \\
\end{array}$ & 0,392 \\
\hline $\begin{array}{l}\text { Cualquier tipo } \\
\text { de progresión }\end{array}$ & & $\begin{array}{l}10,39 \\
72,9 \% \\
55,7 \%\end{array}$ & $\begin{array}{c}6,88 \\
86,2 \% \\
45,3 \%\end{array}$ & $\begin{array}{c}8,51 \\
76,7 \% \\
64,5 \%\end{array}$ & $\begin{array}{c}8,64 \\
74,9 \% \\
63,4 \%\end{array}$ & $\begin{array}{c}17,97 \\
59,2 \% \\
49,4\end{array}$ & 0,083 \\
\hline
\end{tabular}

TPA = Tasa de progresión ajustada por tiempo de seguimiento.

\section{TABLA IIc}

PROBABILIDAD DE PROGRESIÓN DE LA ENFERMEDAD (ANÁLISIS KAPLAN-MEIER). RESULTADOS SEGÚN CIFRAS DE PSA AL DIAGNÓSTICO

\begin{tabular}{|c|c|c|c|c|c|}
\hline & & \multicolumn{3}{|c|}{ Tacto recal } & \multirow{2}{*}{$\begin{array}{c}\text { Log-rank } \\
\text { (p) }\end{array}$} \\
\hline & & Toda la serie & Tumor palpable & No palpable & \\
\hline $\begin{array}{l}\text { Progresión } \\
\text { bioquimica }\end{array}$ & \multirow{4}{*}{$\begin{array}{l}\text { TPA (eventos / } 100 \text { varones x año) } \\
\% \text { libre de progresión a los } 2 \text { años } \\
\% \text { libre de progresión a los } 5 \text { años }\end{array}$} & $\begin{array}{c}9,22 \\
76,8 \% \\
57,9 \% \\
\end{array}$ & $\begin{array}{r}12,23 \\
70,3 \% \\
55,2 \% \\
\end{array}$ & $\begin{array}{c}7,92 \\
80,4 \% \\
59,9 \% \\
\end{array}$ & 0,111 \\
\hline $\begin{array}{l}\text { Progresión } \\
\text { clínica }\end{array}$ & & $\begin{array}{c}3,28 \\
91,3 \% \\
85,0 \%\end{array}$ & $\begin{array}{c}4,52 \\
88,9 \% \\
70,2 \%\end{array}$ & $\begin{array}{c}2,63 \\
92,4 \% \\
92,4 \%\end{array}$ & 0,223 \\
\hline $\begin{array}{l}\text { Progresión a } \\
\text { enfermedad } \\
\text { metastásica }\end{array}$ & & $\begin{array}{c}1,20 \\
96,0 \% \\
94,5 \%\end{array}$ & $\begin{array}{c}2,01 \\
91,9 \% \\
86,9 \%\end{array}$ & $\begin{array}{c}0,79 \\
98,1 \% \\
98,1 \%\end{array}$ & 0,170 \\
\hline $\begin{array}{l}\text { Cualquier tipo } \\
\text { de progresión }\end{array}$ & & $\begin{array}{l}10,39 \\
72,9 \% \\
55,7 \%\end{array}$ & $\begin{array}{l}12,58 \\
68,6 \% \\
53,7 \%\end{array}$ & $\begin{array}{c}9,38 \\
75,3 \% \\
57,1 \%\end{array}$ & 0,242 \\
\hline
\end{tabular}

TPA $=$ Tasa de progresión ajustada por tiempo de seguimiento.

TABLA IId

PROBABILIDAD DE PROGRESIÓN DE LA ENFERMEDAD (ANÁLISIS KAPLAN-MEIER) RESULTADOS SEGÚN SCORE DE GLEASON EN LA BIOPSIA

\begin{tabular}{|c|c|c|c|c|c|c|c|}
\hline & & \multicolumn{5}{|c|}{ PSA al diagnóstico (ng/ml) } & \multirow{2}{*}{$\begin{array}{l}\text { Log-rank } \\
\text { (p) }\end{array}$} \\
\hline & & Toda la serie & $2-4$ & 4-5 & 7 & $8-10$ & \\
\hline $\begin{array}{l}\text { Progresión } \\
\text { bioquímica }\end{array}$ & \multirow{4}{*}{$\begin{array}{l}\text { TPA (eventos / } 100 \text { varones x año) } \\
\% \text { libre de progresión a los } 2 \text { años } \\
\% \text { libre de progresión a los } 5 \text { años }\end{array}$} & $\begin{array}{c}9,22 \\
76,8 \% \\
57,9 \%\end{array}$ & $\begin{array}{l}12,05 \\
68,7 \% \\
51,0 \%\end{array}$ & $\begin{array}{c}6,41 \\
83,3 \% \\
70,4 \%\end{array}$ & $\begin{array}{l}12,87 \\
66,8 \% \\
47,7 \%\end{array}$ & $\begin{array}{c}3,77 \\
88,2 \% \\
88,2 \%\end{array}$ & 0,134 \\
\hline $\begin{array}{l}\text { Progresión } \\
\text { clínica }\end{array}$ & & $\begin{array}{c}3,28 \\
91,3 \% \\
85,0 \%\end{array}$ & $\begin{array}{c}3,28 \\
92,2 \% \\
85,6 \%\end{array}$ & $\begin{array}{c}3,08 \\
91,5 \% \\
87,6 \%\end{array}$ & $\begin{array}{c}2,41 \\
96,6 \% \\
96,6 \%\end{array}$ & $\begin{array}{l}13,13 \\
66,7 \% \\
47,6 \%\end{array}$ & 0,022 \\
\hline $\begin{array}{l}\text { Progresión a } \\
\text { enfermedad } \\
\text { metastásica }\end{array}$ & & $\begin{array}{c}1,20 \\
96,0 \% \\
94,5 \%\end{array}$ & $\begin{array}{c}1,64 \\
94,3 \% \\
94,3 \%\end{array}$ & $\begin{array}{l}\text { No eventos } \\
100 \% \\
100 \%\end{array}$ & $\begin{array}{l}\text { No eventos } \\
100 \% \\
100 \%\end{array}$ & $\begin{array}{l}13,13 \\
66,7 \% \\
47,6 \%\end{array}$ & $<0,001$ \\
\hline $\begin{array}{l}\text { Cualquier tipo } \\
\text { de progresión }\end{array}$ & & $\begin{array}{c}10,39 \\
72,9 \% \\
55,7 \%\end{array}$ & $\begin{array}{l}12,68 \\
68,2 \% \\
50,6 \%\end{array}$ & $\begin{array}{c}8,02 \\
78,0 \% \\
68,2 \%\end{array}$ & $\begin{array}{c}11,40 \\
70,4 \% \\
50,3 \%\end{array}$ & $\begin{array}{l}14,39 \\
56,0 \% \\
56,0 \%\end{array}$ & 0,339 \\
\hline
\end{tabular}

TPA = Tasa de progresión ajustada por tiempo de seguimiento. 


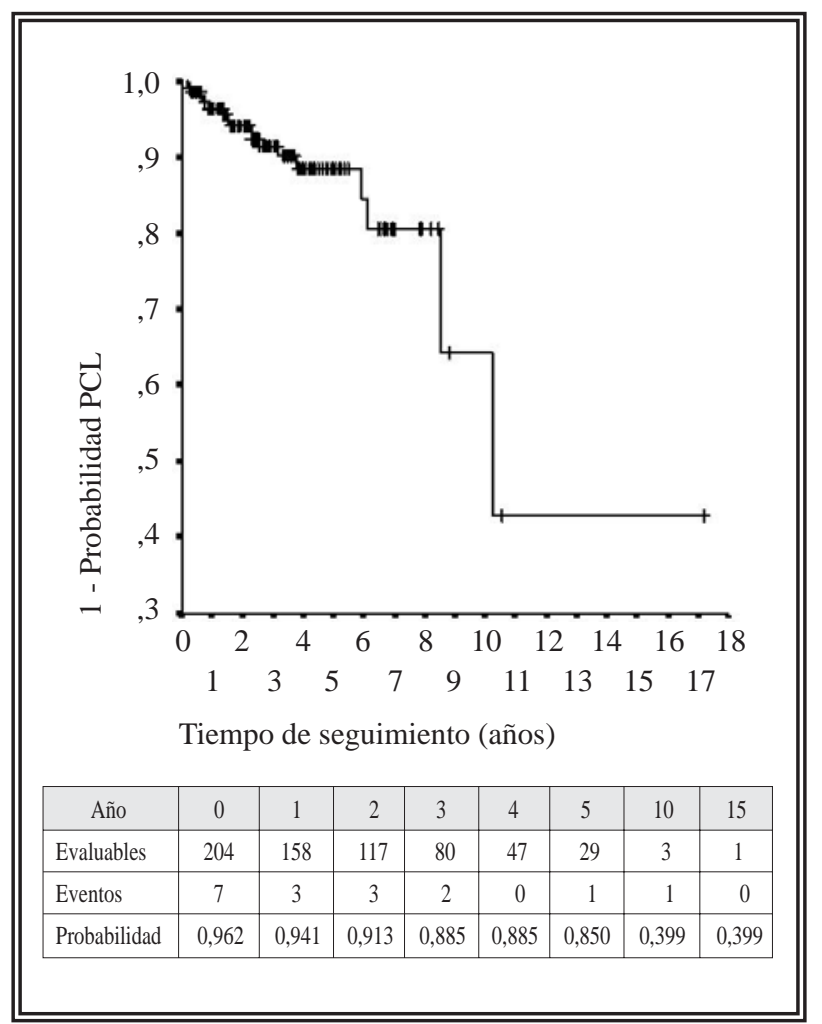

FIGURA 2a. Curva Kaplan-Meier que muestra la probabilidad de toda la serie de permanecer libre de progresión clinica.

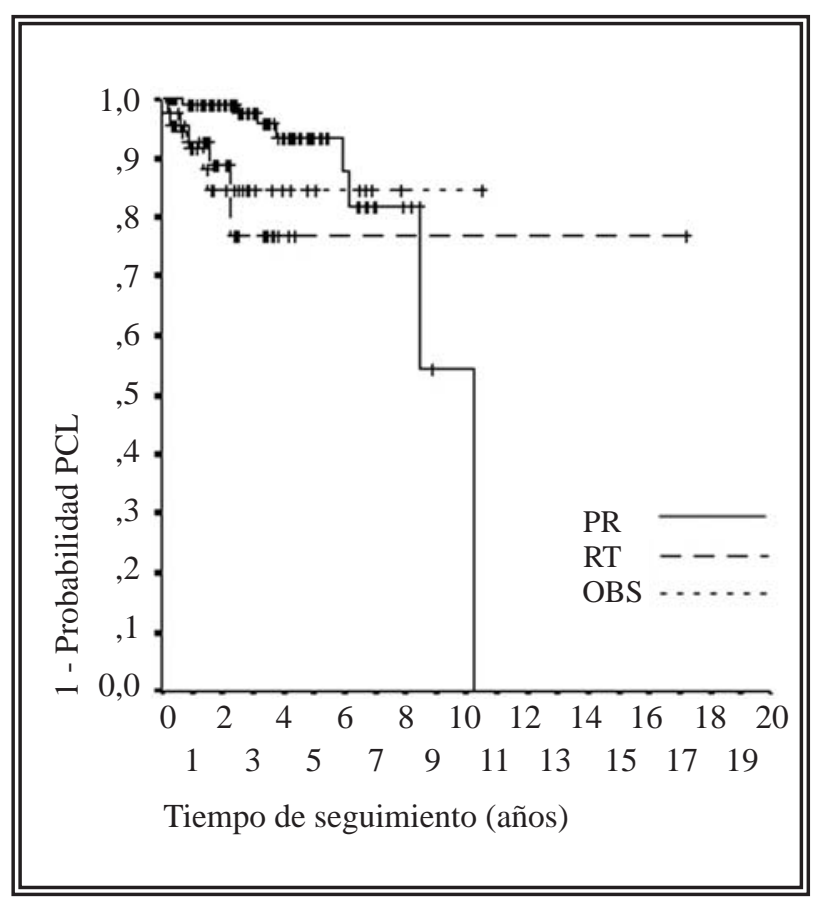

FIGURA 2b. Comparación de los grupos de tratamiento estudiado respecto del evento progresión clinica. Test log-rank $p=0,109$. $(P R=$ prostatectomia radical, $R T=$ radioterapia, $\mathrm{OBS}$ = observación).

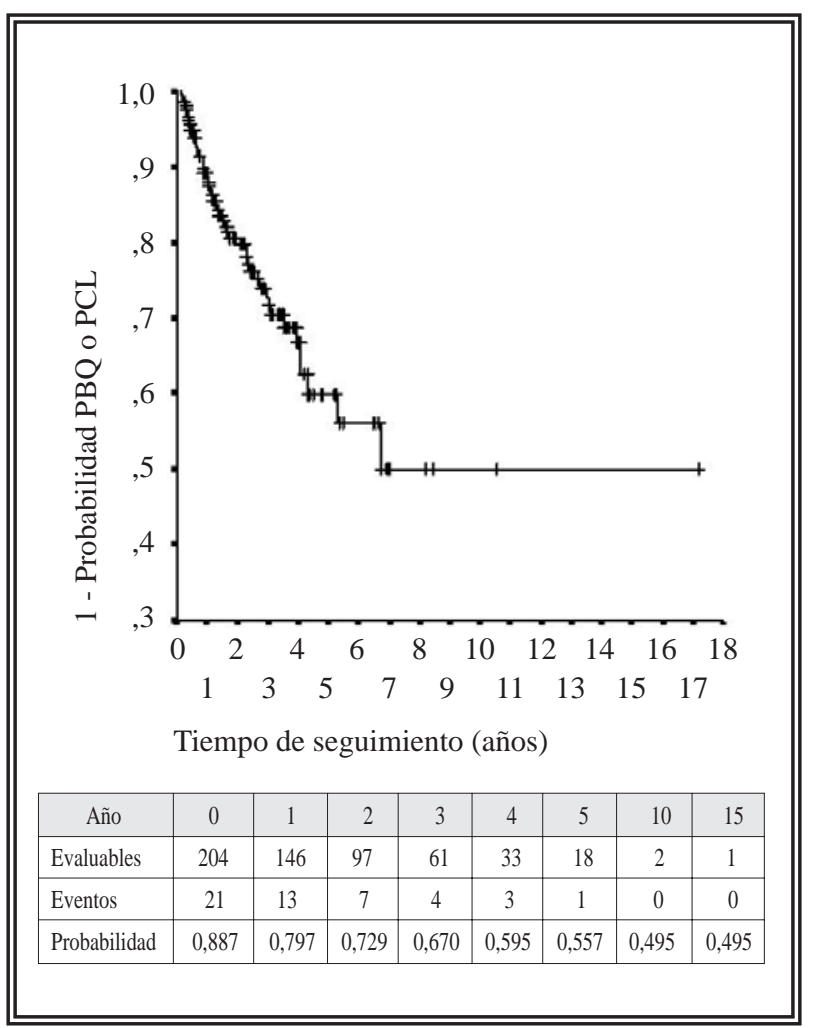

FIGURA 3a. Análisis actuarial de toda la serie donde se estudia la probabilidad de presentar cualquier tipo de progresión (bioquímica, clínica o metastásica -lo que primero suceda-).

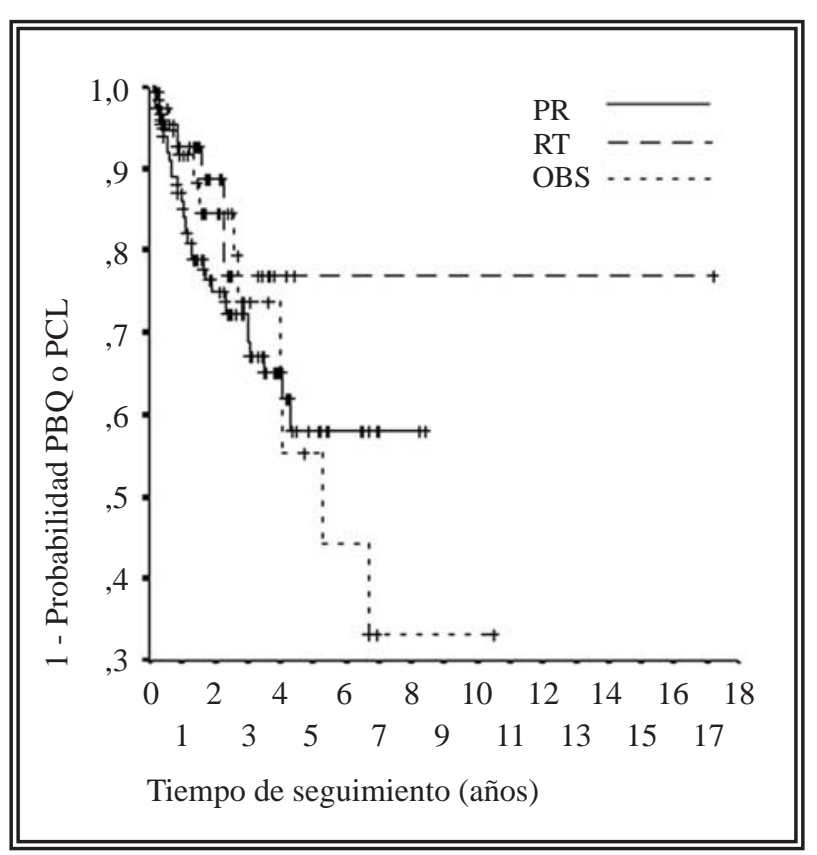

Figura 3b. Comparación entre los grupos de tratamiento de este estudio con respecto a la probabilidad de presentar cualquier tipo de progresión (bioquimica, clinica o metastásica -lo que primero suceda-). Test logrank $p=0,470$. 
TABLA III

SUPERVIVENCIA GLOBAL MEDIA (NO EXISTIÓ MORTALIDAD CÁNCER-ESPECÍFICA)

\begin{tabular}{|c|c|c|c|c|}
\hline & & \multicolumn{2}{|c|}{ Supervivencia global } & \multirow[b]{2}{*}{ Log-rank (p) } \\
\hline & & 2 años & 5 años & \\
\hline Todos los casos & & $96,5 \%$ & $95,1 \%$ & \\
\hline \multirow{6}{*}{ Edad años } & $41-45$ & \multicolumn{2}{|c|}{ No eventos (100\%) } & 0,368 \\
\hline & $46-50$ & \multicolumn{2}{|c|}{ No eventos (100\%) } & \\
\hline & $51-55$ & \multicolumn{2}{|c|}{ No eventos (100\%) } & \\
\hline & $56-60$ & \multicolumn{2}{|c|}{ No eventos (100\%) } & \\
\hline & $61-65$ & $91,5 \%$ & $91,5 \%$ & \\
\hline & $66-70$ & $92,5 \%$ & $87,7 \%$ & \\
\hline \multirow{3}{*}{ Tratamiento } & PR & $99,2 \%$ & $99,2 \%$ & 0,034 \\
\hline & RT & $96,5 \%$ & $85,8 \%$ & \\
\hline & OBS & $87,7 \%$ & $87,7 \%$ & \\
\hline \multirow{4}{*}{$\begin{array}{l}\text { PSA inicial } \\
\text { (ng/ml) }\end{array}$} & $<4$ & $95,0 \%$ & $95,0 \%$ & 0,289 \\
\hline & $\geq 4 \mathrm{y}<7$ & $91,4 \%$ & $91,4 \%$ & \\
\hline & $\geq 7 y<10$ & \multicolumn{2}{|c|}{ No eventos (100\%) } & \\
\hline & $\geq 10$ & $100 \%$ & $95,2 \%$ & \\
\hline \multirow[t]{2}{*}{ Tumor palpable } & $\mathrm{TR}+$ & $98,5 \%$ & $98,5 \%$ & 0,400 \\
\hline & TR - & $95,6 \%$ & $93,6 \%$ & \\
\hline \multirow[t]{4}{*}{ Gleason score } & $2-4$ & $91,5 \%$ & $91,5 \%$ & 0,317 \\
\hline & $5-6$ & $98,9 \%$ & $98,9 \%$ & \\
\hline & 7 & $95,7 \%$ & $87,3 \%$ & \\
\hline & $8-10$ & \multicolumn{2}{|c|}{ No eventos (100\%) } & \\
\hline
\end{tabular}

La tasa de permanecer libre de progresión a los 5 años tras la PR oscila, en la mayoría de las series, entre el 77 y $80 \%{ }^{2}$. Hull et al. obtuvieron $78 \%$ de pacientes libres de $\mathrm{PBQ}$ a los 5 años desde la $\mathrm{PR}^{10}$. En nuestra serie este dato ha sido notablemente inferior $(57,6 \%)$, aunque es necesario tener en cuenta el corto seguimiento, ya que únicamente 12 pacientes fueron evaluables tras dicho periodo.

Similares resultados pueden encontrarse en las series donde se evalúa el control del CaP mediante RT. Fukunaga-Johnson y cols. encontraron un 75\% de pacientes libres de PBQ a los 5 años en pacientes con rasgos clínicos favorables (37\% si datos desfavorables: T3-T4, Gleason score $>7$, o PSA $>10 \mathrm{ng} / \mathrm{mL})^{11}$. Nosotros hemos obtenido un 100\% libres de PBQ a los 5 años, y un $76,8 \%$ de cualquier progresión. De nuevo el corto seguimiento (2 pacientes evaluables para PBQ tras ese periodo, y 1 paciente para progre- sión de todo tipo) hace poco viable cualquier comparación.

A nuestro juicio, las diferencias encontradas en las tasas de PBQ entre las modalidades de tratamiento estudiadas en nuestra serie (favoreciendo de modo significativo la RT y penalizando la PR) no merecen ser consideradas. Esto se debe a la facilidad de catalogar la PBQ tras la PR (es suficiente con una sola determinación de PSA detectable), mientras que para encontrar una PBQ en los grupos RT y OBS es necesario disponer de al menos cuatro determinaciones de PSA. Teniendo en cuenta que en nuestro Centro las revisiones en pacientes clinicamente estables se realizan de modo semestral o anual, no es de extrañar que sea mucho más fácil diagnosticar una $\mathrm{PBQ}$ en los pacientes sometidos a PR (donde tan sólo bastaría una revisión con PSA >0,2 $\mathrm{ng} / \mathrm{ml}$ ) que a los que optaron por las otras dos alternativas estudiadas. 


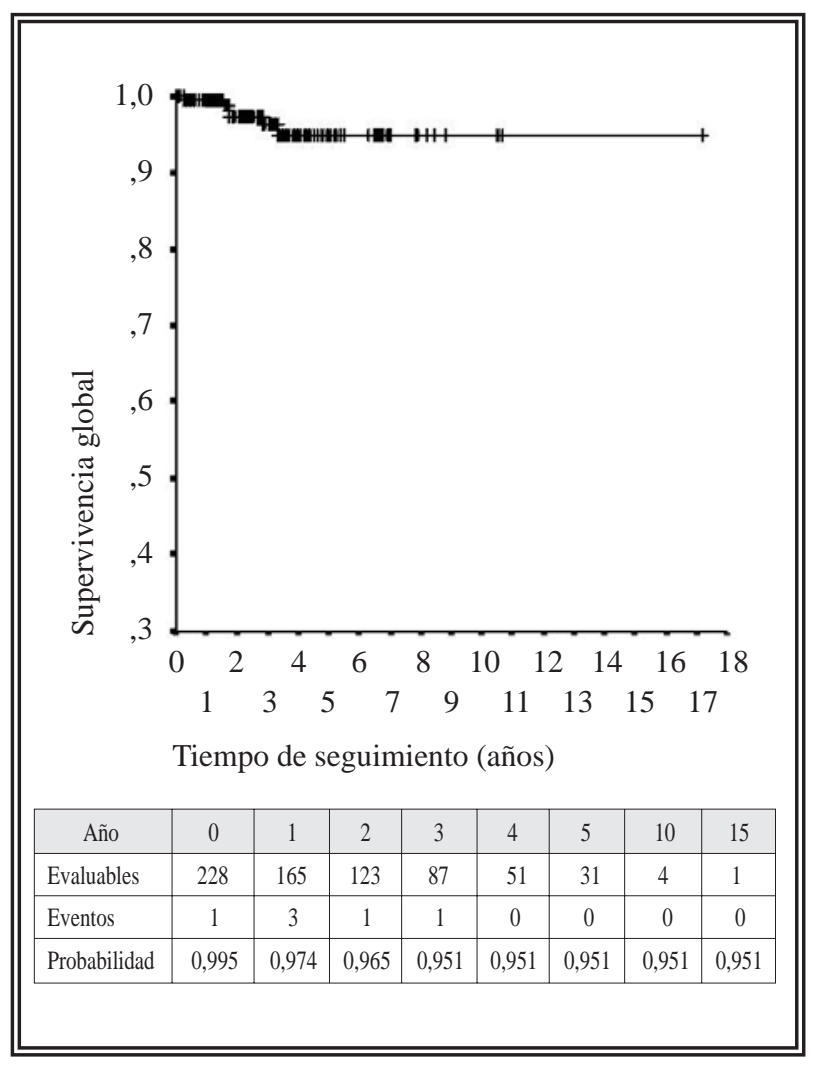

FIGURA 4a. Probabilidad de supervivencia global en toda la serie.

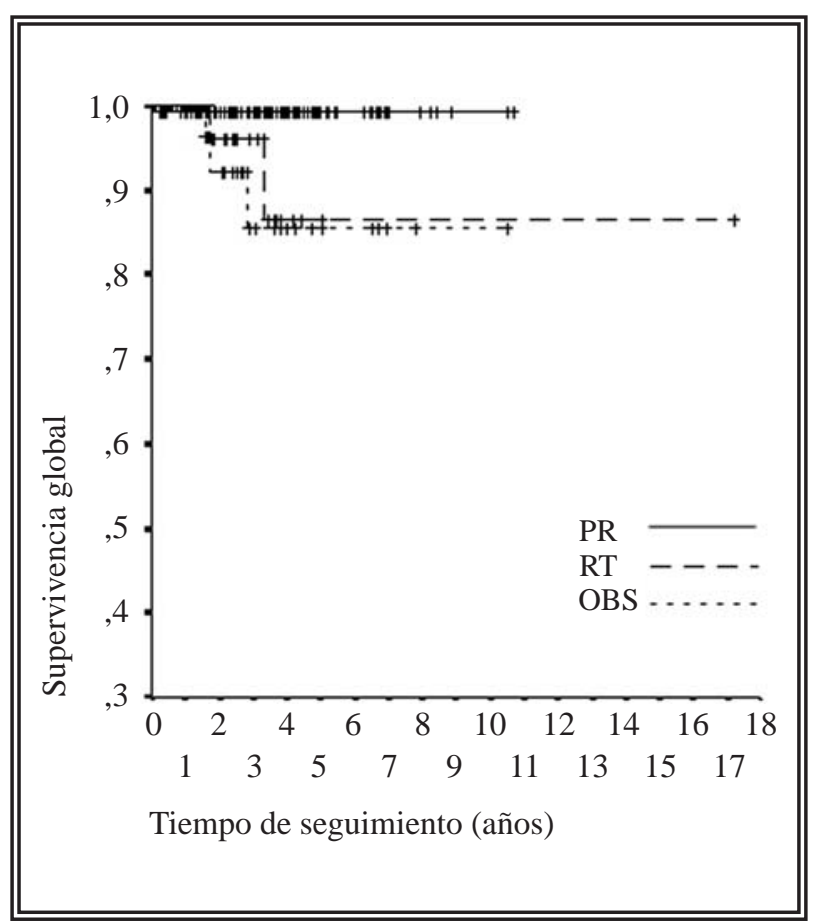

FIGURA 4b. Comparación entre los grupos de tratamiento con respecto a la supervivencia global. Test logrank $p=0,034$.
En un estudio randomizado en el que se compararon (estudiando progresión y supervivencia) PR y OBS, se encontró que la tasa de progresión de la enfermedad fue superior en los pacientes no sometidos a tratamiento curativo ${ }^{7}$. En cuanto a la mortalidad, se evidenció un aumento significativo de la supervivencia libre de enfermedad tras la cirugía frente a la vigilancia, aunque sin impacto en la supervivencia global. Por tanto, el debate respecto de la conveniencia o no del tratamiento agresivo de esta enfermedad sigue abierto. No obstante, en nuestro estudio las cifras de progresión del grupo OBS son similares a la de pacientes sometidos a PR y además, debido a la "juventud" de nuestra serie, aún no ha sido posible registrar mortalidad por CaP. McLaren y cols. obtuvieron una supervivencia global a los 2 y 5 años de $92 \%$ y $68 \%$, respectivamente ${ }^{12}$. En nuestra serie, la supervivencia global sin tratamiento fue de $87,7 \%$ tanto a los 2 como a los 5 años, sin diferencia significativa respecto a las opciones de terapia activa. En cualquier caso, el hecho de no existir aún en nuestra serie mortalidad cáncer específica limita de modo considerable cualquier especulación en términos de supervivencia. Un ejemplo es la ventaja en cuanto a supervivencia obtenida por la PR respecto de las otras dos opciones. Debido a la ausencia de mortalidad cáncer-específica, dicha diferencia debe atribuirse a factores distintos al tratamiento, como por ejemplo a una selección de pacientes con menor comorbilidad, etc.

No obstante, aunque no hayamos evidenciado todavía mortalidad por $\mathrm{CaP}$ en esta serie, no conviene olvidar que un $3,4 \%$ de nuestros pacientes progresó a enfermedad metastásica y que este tipo de progresión sufrió una mayor tendencia (no significativa) entre los pacientes sometidos a OBS $(2,85$ eventos progresión metastásica / 100 varones x año) en comparación con los otros dos grupos terapéuticos (PR 0,79 y RT 1,05). Donde si se mostró diferencia estadística fue al tener en cuenta el score de Gleason, donde se registraron 13,13 eventos progresión metastásica / 100 varones $\mathrm{x}$ año en el grupo con score 8-10 en comparación con el total de la serie $(1,20, \mathrm{p}<0,001)$. 


\section{CONCLUSIONES}

Aunque los datos de nuestra serie son preliminares, podemos concluir que el pronóstico a corto y medio plazo del cáncer de próstata localizado es, en términos de supervivencia, excelente con o sin tratamiento activo en pacientes con suficiente esperanza de vida. No obstante, aunque la mortalidad cáncer-específica es excepcional durante los primeros años del seguimiento, algunos enfermos pueden desarrollar enfermedad metastásica, riesgo que aumenta si se opta por la vigilancia (tendencia no significativa), así como en pacientes con score de Gleason pobremente diferenciado.

Agradecimientos: Al Dr. Carlos Llorente en especial y al resto de facultativos por su intervención en el mantenimiento de esta base de datos a su paso por nuestro Departamento.

A Open Group Development, compañia que colaboró en la puesta en marcha de la nueva base de datos integral de nuestro Servicio.

Nuestro Departamento es Miembro del European Randomized Study of Screening for Prostate Cancer (ERSPC).

Financiado en parte por becas del Fondo de Investigación Sanitaria (93/093, 96/0248, 96/ 1800, 99/0245, 02/0732).

\section{REFERENCIAS}

1. http://www.ine.es (último acceso 12 de Abril de 2003).

2. EASTHAM JA, SCARDINO PT.: Radical prostatectomy. En Campbell's Urology 4를. pp: 3080-3106. W.B. Saunders, Philadelphia, PA, USA. 2002.

3. CARTER HB, PARTIN AW.: Diagnosis and staging of prostate cancer. En Campbell's Urology 4⿳亠丷厂犬Ed. pp: 3055-3079. W.B. Saunders, Philadelphia, PA, USA. 2002.
4. CHODAK GW, THISTED RA, GERBER GS et al.: Results of conservative management of clinically localized prostate cancer. $N$ Engl $J$ Med 1994; 330: $242-248$.

5. ALBERTSEN PC, FRYBACK DG, STORER BE, KOLON TF: Long-term survival among men with conservatively treated localized prostate cancer. JAMA 1995; 274: 626-631.

6. JOHANSSON JE, HOLMBERG L, JOHANSSON S, BERGSTROM R, ADAMI HO: Fifteen-year survival in prostate cancer. A prospective, population-based study in Sweden. JAMA 1997; 277: 467-471.

7. HOLMBERG L, BILL AXELSON A, HELGESEN F, et al: A randomized trial comparing radical prostatectomy with watchful waiting in early prostate cancer. N Engl J Med 2002; 347(11): 781-9.

8. ALBERTSEN PC, HANLEY JA, GLEASON DF, BARRY MJ: Competing risk analysis of men aged 55 to 74 years at diagnosis managed conservatively for clinically localized prostate cancer. JAMA 1998; 280: 975-980.

9. COX JD: The American Society for Therapeutic Radiology and Oncology Consensus Panel. Consensus Statement Guidelines for PSA Failure Following Radiation Therapy. Int J Radiat Oncol Biol Phys 1997; 37: 1.035-1.041.

10. HULL GW, RABBANI F, ABBAS F, WHEELER TM, KATTAN MW, SCARDINO PT: Cancer control with radical prostatectomy alone in 1000 consecutive cases. J Urol 2002; 167: 528-534.

11. FUKUNAGA JOHNSON N, SANDLER HM, MCLAUGHLIN PW, et al: Results of 3D conformal radiotherapy in the treatment of localized prostate cancer. Int J Radiat Oncol Biol Phys 1997; 38: 311-317.

12. MCLAREN DB, MCKENZIE M, DUNCAN G, PICKLES T: Watchful waiting or watchful progression?: Prostate specific antigen doubling times and clinical behavior in patients with early untreated prostate carcinoma. Cancer 1998; 82(2): 342-8.

Dr. Marcos Luján

Servicio de Urología. Hospital Univ. de Getafe

Ctra. Toledo km. 12,500

28905 Getafe (Madrid)

marcoslujan@terra.es

(Trabajo recibido el 25 julio 2003) 